\title{
Städte als Innovationszentren für eine Neubesinnung ethnischer Minderheiten. Das Beispiel der Kärntner Slowenen (Österreich)
}

\section{Ernst Steinicke, Innsbruck}

\section{Vorbemerkungen und These}

Wie aus Immigrationsstudien und kontaktlinguistischen Arbeiten ableitbar ist (HAARMANn 1983, NELDE 1987, Glebe \& O’Loughlin 1987, Fassmann \& MüNZ 1994), sind ethnische Minderheiten in Verdichtungsräumen besonderen Einflussfaktoren ausgesetzt. Die Übernahme städtischer Verhaltens- und Handlungsmuster, die Vereinheitlichung von Lebensstilen sowie die große Anzahl an sprachlich gemischten Ehen hat letztendlich eine assimilationsfördernde Wirkung, die in der Regel dem staatstragenden Volk zugute kommt. So ist es verständlich, dass autochthone ethnische Minderheiten (nationale Minderheiten) in Städten im Gefolge der Industrialisierung mehr und mehr verschwinden. Dagegen können sich solche Gruppen in ländlichen Räumen, v.a. in peripher gelegenen Gebieten, bis in die Gegenwart herein vergleichsweise gut halten - als «verbleibende Konsequenz traditioneller, räumlich und zeitlich verankerter Lebensformen» (WERLEN 2000: 343).

Der vorliegende Beitrag geht von der These aus, dass im Einfluss der postindustriellen Entwicklung sowie mit zunehmendem Minderheitenschutz Verdichtungsräume einen Funktionswandel erleben: Städte erweisen sich immer mehr als Impulsgeber für das Neuerwachen von ethnischen Identitäten bzw. die Wiederbelebung des Selbstverständnisses von Minderheiten. Zwar bestehen die Ungunstfaktoren für die Bewahrung autochthoner ethnischer Gruppen in Agglomerationen weiterhin, doch werden sie überlagert von neuen Entwicklungen, die geeignet sind, die Ethnodiversität zu bewahren. Diese vom städtischen Raum diffusionsartig ausgehenden Impulse zur ethnischen Neubesinnung erfassen in der Folge auch die Siedlungsgebiete ethnischer Minoritäten der Peripherie. Als mittelfristige Konsequenz lässt sich daraus für das westliche Europa ein Abschwächen der territorialen und Assimilationsverluste von Minderheiten ableiten.

Bevor diese Gedanken theoretisch näher erläutert und empirisch am Fallbeispiel der Kärntner Slowenen veranschaulicht werden, erscheint es notwendig, den bereits mehrfach verwendeten Begriff «ethnische Minderheit» zu umreißen. Heckmann (1978, 1992), HaARmann (1983), KraAs-Schneider (1989), KraAs (1992b), Fenet et al. (1995) und Plasseraud (1998) zeigen die
Schwierigkeit auf, «Minderheit» befriedigend zu definieren. Einmal wird darunter eine zahlenmäßig unterlegene Bevölkerungsgruppe verstanden, sehr oft aber auch eine nicht-dominierende, sozial benachteiligte Gruppe. Sie ist sich ihrer Stellung bewusst und besitzt ein Gemeinschaftsgefühl als Gruppe.

Unmöglich ist es jedoch, eine allgemein gültige Definition von «ethnische Minderheiten» zu liefern (Mühlmann 1964, Barth 1969, Kloss 1969, Breton 1983, Heckmann 1978, 1992, Veiter 1984, Héraud 1993, Koter 1993, Käufeler 1994, Pan \& Pfeil 2000). Hier unterscheidet sich die Sprachpraxis oftmals von Staat zu Staat. Beispielsweise werden in weiten Teilen Europas ethnische Minderheiten als Sondergruppen innerhalb eines Staates bezeichnet, die sich durch objektive (v.a. kulturelle) und subjektive (Gemeinschaftsgefühl) Faktoren vom Mehrheitsvolk unterscheiden. Voraussetzung ist zudem ein gemeinschaftlicher Wille, die Sonderstellung zu erhalten, sowie eine gewisse autochthone Situation, was eine Ansässigkeit von mindestens drei Generationen erfordert (VEITER 1984). So bilden in Mitteleuropa Gastarbeiter und Immigranten aus der Türkei oder aus Südosteuropa zwar ethnische Gruppen, sie werden aber nicht als «ethnische Minderheiten» bezeichnet. In den Niederlanden, den USA, in Großbritannien und Kanada besteht demgegenüber eine völlig andere Sichtweise, in der Immigranten durchaus zur Kategorie «ethnische Minderheiten» zählen können (PLETSCH 1985, U.S. Department of Commerce 1990, EntzinGer 1998, Fassmann \& Münz 1994, CBS 1999, SteiNICKE 2001a). Einen Sonderfall bildet Frankreich, das offiziell auf seinem Staatsgebiet keine ethnischen Minderheiten kennt - sieht man einmal von der de facto-Situation bei den Korsen ab (SANGUIN 1993, Meistersheim 1999, 2001).

Den folgenden Ausführungen liegt das mitteleuropäische Begriffsverständnis zugrunde (STEINICKE 2001a). Die Bodenständigkeit der sprachlichen Sondergruppen (autochthone ethnolinguistische Gruppen) spielt demnach die zentrale Rolle bei der Definition ethnischer Minderheiten.

Ziel dieser Studie ist es, den Einfluss städtischer Akteure auf den Erhalt der ethnischen Minoritäten im westlichen Europa aufzuzeigen. Die oben erwähnte These soll anhand des aktuellen Forschungsstandes sowie der Interpretation von Primärquellen (amtliche Statistiken, eigene Erhebungen) theoretisch wie auch 
empirisch überprüft und gegebenenfalls auf die Ebene eines theoretischen Ansatzes gebracht werden.

\section{Theoretischer Rahmen}

\subsection{Ethnische Territorialentwicklung und Assimilation}

Aufgrund des zahlenmäßigen Kräfteverhältnisses zwischen Majorität und Minorität lassen sich prinzipiell drei Grundtypen der Territorialentwicklung von ethnischen Minderheiten erkennen (vgl. KraAS 1992a: 315, vgl. dazu auch SteINICKE 1995: 53):

- ein im Laufe der Zeit konstantes Territorium,

- ein wachsendes Territorium,

- ein sich zurückbildendes Territorium («Territorialregression»).

Für die meisten Minderheiten ist eine Territorialregression mit einherschreitender Abnahme der Zahl der Minderheitsangehörigen bezeichnend. Diese Einengung von Siedlungsgebieten wird einerseits durch Assimilation an die Majorität - also v.a. durch den
Sprachwechsel - verursacht, zum zweiten können dafür ebenso demographische Veränderungen der betreffenden Gruppe, wie zum Beispiel Migrationen oder Geburtendefizite, verantwortlich sein. Berücksichtigt man die zeitliche Dimension, so verändert sich das Grundmuster dafür folgendermaßen (vgl. Abb. 1):

- Die erste Phase ist durch die Einengung des Siedlungsgebietes geprägt. Dies macht sich einerseits in einem allgemeinen Ausdünnungsprozess, d.h. in der abnehmenden Zahl von Minderheitsangehörigen bemerkbar, zum anderen kommt es zur räumlichen Peripherisierung:Zentrumsnahe und verkehrsmäßig gut erreichbare Siedlungen werden assimiliert, in den isolierten, abgeschiedenen Gegenden (Passivräumen) bleibt die Minderheit dagegen erhalten. Bei nationalen Minderheiten, also solchen Gruppen, die ihr kulturelles Mutterland im Nachbarstaat haben und deren Siedlungsgebiete in der Regel am Rand eines Staates liegen, verlagert sich das Minderheitenterritorium damit verstärkt in Richtung Staatsgrenze (Phase 1).

- Sieht man von Zwischenstadien ab, so lässt sich eine zweite Phase mit dem Wandel vom geschlosse-

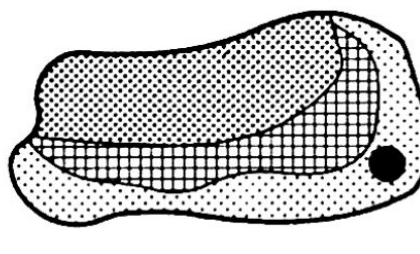

Phase 1

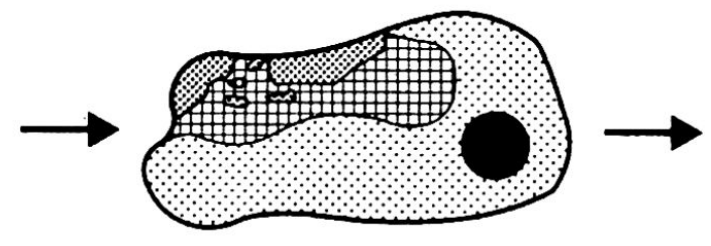

Phase 2

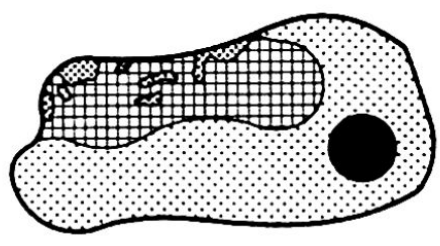

Phase 3

\section{Stadien der \\ Territorialregression}

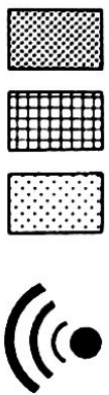

20./21. Jhdt.

frühere Stadien

grösste

Ausdehnung

Einfluss

urbaner Raum

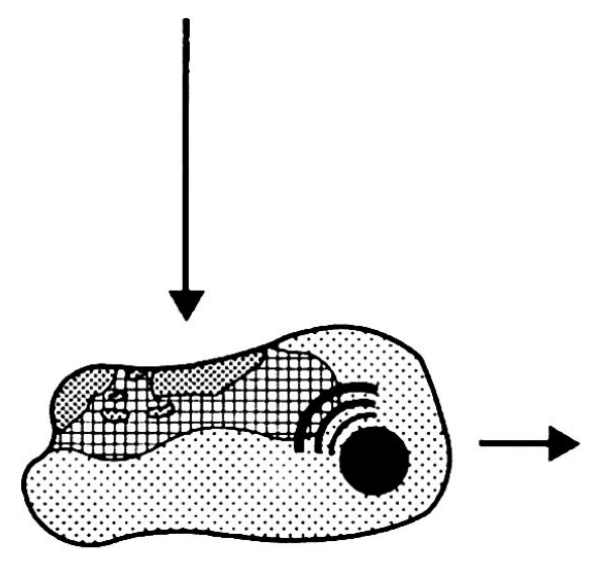

Phase $2^{1}$

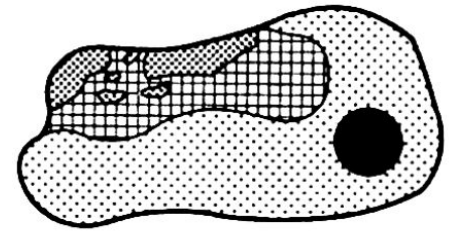

Phase $3^{\prime}$

Abb. 1: Prozess der Einengung des Siedlungsgebietes von ethnischen Minderheiten

Phases of territorial regression of ethnic minorities

Phases de régression territoriale des minorités ethniques

Entwurf: E. STEINICKE, Bearbeitung: L. BaumanN 
nen Siedlungsgebiet zu dispersen Gebieten, evtl. mit einem Zentralbereich, erkennen.

- Am Ende der Entwicklung liegen reliktäre Gebiete vor, von denen später nur mehr historische Reminiszenzen übrigbleiben (Phase 3).

- Sollte allerdings die hier vorgebrachte Leitthese verifiziert werden (Phase 2'), so würde sich zwangsläufig auch die rückläufige Tendenz ändern. Wie im Schema angedeutet, folgt in diesem Fall eine Phase der Konstanz bzw. sogar Expansion des Siedlungsgebietes von Minderheiten (Phase 3').

Eine Sprache stirbt dann, wenn sie die Kinder nicht mehr sprechen. Zentrale Bedeutung für ihre Erhaltung oder umgekehrt für ihre Zurückdrängung haben demnach Kindergarten und Schule sowie die weiteren kulturpolitischen Instrumente der staatstragenden Mehrheit, wie etwa Medien oder Verwaltungssprache. Daneben erfolgte der Einfluss der Majorität auf die Minorität vor allem über die kulturellen Traditionen des Mehrheitsvolks sowie über die Wirtschaft. Weitere Faktoren, die eine Bereitschaft zur Assimilation erhöhen oder verringern können, sind zum Beispiel Verkehrserschließung und Kommunikation, die Lage zum kulturellen Kernland, Sprachverwandtschaft, dialektale Aufsplitterung, Kodifizierungsstand der Schriftsprache sowie moderne Trends in Sport, Mode und Musik. Wie an anderer Stelle ausgeführt (STEINICKE 1991: 13-17), ist aber für die endgültige Assimilation der jüngeren Generation letztlich die sprachliche Herkunft der Erziehenden, also der Elternteile, entscheidend.

Von zentraler Bedeutung für die ethnische Selbstbehauptung und damit für ein Zurückdrängen der Assimilation ist das Ausbildungs- und Qualifikationsniveau. Als gesichert gilt, dass das ethnische Bewusstsein generell mit zunehmendem Ausbildungsgrad ansteigt. Es verwundert daher nicht, wenn in der bildungsgeographischen Literatur die wirtschaftliche und soziale Entwicklung von Minoritäten sowie das ethnische Selbstverständnis eng mit der Schulfrage verbunden wird (Meusburger 1979, 1980: 144-149, 1996, 1998 : 354-367, WARREN 1982, ERIKSEN et al. 1991, TomiaK \& Kazamias 1991). Auch in vorliegender Studie nimmt dieser Aspekt eine Schlüsselposition ein, wenngleich hier nicht der Platz ist, auf das allgemeine Konfliktpotenzial Minderheit und Schulpolitik einzugehen.

Hervorzuheben ist die bildungsgeographische Erkenntnis, dass ethnische Minderheiten häufig entweder im unteren oder im oberen Bereich der Ausbildungshierarchie überdurchschnittlich vertreten sind (MEUSBURGER 1998: 355). Dies steht zuweilen im Widerspruch zum Alltagsverständnis, das Minoritäten grundsätzlich an die Basis der sozialen Schichtung stellt. MeusBURGER (1998: 357) erklärt ein auffallend hohes Aus- bildungsniveau von Minderheiten, wie es beispielsweise für Chinesen und Japaner in den Vereinigten Staaten typisch ist, durch eine besondere Leistungsbereitschaft v.a. aufgrund drohender Diskriminierung. Dem ist noch hinzuzufügen, dass ethnische Minderheiten bei toleranter und aufgeschlossener Kulturpolitik auch von staatlicher Seite profitieren können: Wie in den späteren Kapiteln aufgezeigt wird, kann sich mit der Errichtung von Bildungsstätten, die spezifisch auf die kulturellen Bedürfnisse der Minorität ausgerichtet sind, in raschem Tempo ihr Ausbildungs- und Qualifikationsniveau anheben.

\subsection{Periphere Minderheit und sozialräumliche Entwicklung}

Versteht man unter Periphergebieten Raumeinheiten, die in sozialer, wirtschaftlicher und infrastruktureller Hinsicht benachteiligt sind, so lassen sich zahlreiche Minoritäten in Europa aufzählen, die in solcherart strukturierten Regionen leben. Ein Blick auf die ethnische Landkarte des westlichen Europa vermittelt zudem, dass die meisten dieser Gruppen ihre Siedlungsgebiete in räumlicher Distanz vom wirtschaftlichen und administrativen Hauptzentrum eines Staates haben. Wirtschaftliche Marginalität fällt mit peripherer Lage zusammen. Das gilt beispielsweise für die Galicier in Spanien, die Sorben in Deutschland oder für alle ungeschützten, bodenständigen ethnolinguistischen Minderheiten in Italien, Frankreich und Großbritannien. Auch die drei bekannten Minoritätengruppen Österreichs, die Slowenen, Kroaten und Ungarn, sind am Rand des Staatsgebietes zu finden.

$\mathrm{Da}$ in den peripheren Regionen die modernen sozioökonomischen Wandlungen verzögert ablaufen, ist es verständlich, dass ethnisch-kulturelle Besonderheiten erhalten bleiben können. Dies wird vor allem in den Siedlungsgebieten kleiner Volksgruppen deutlich. Hier verbindet sich mit der Peripherlage - nicht selten auch naturräumlich verstärkt - eine Abgeschiedenheit und Isolation, welche die Bewahrung der ethnischen Vielfalt unterstützt hat. Mit der zunehmenden Industrialisierung setzten aber auch in solchen abgeschiedenen Lagen letztendlich Migrationen in die Zentralräume ein, welche die Minderheiten quantitativ und qualitativ schwächten und die Assimilation vorantrieben: Ein Gutteil der Minoritäten erlitt damit Bevölkerungs- und Territorialverluste, wobei das Ausmaß von zahlreichen Variablen abhing. Beispielsweise konnten geschlossene Siedlungsweisen oder kulturpolitische Ausnahmeregelungen diesen Prozess abschwächen.

Der gesamtgesellschaftliche Wandel ist allerdings nicht in der Phase der Industrialisierung stehen geblieben. In vielen Teilen Europas wurde diese bereits im Laufe der siebziger Jahre von der Phase der Tertiärisierung 
(postindustrielle Phase) abgelöst. Dieser moderne (bzw. spätmoderne) Strukturwandel hat nun ebenfalls in den Siedlungsgebieten der ethnischen Minderheiten eingesetzt, und Tourismus, Handel und andere Dienstleistungsbranchen sind dort oftmals zu den bedeutendsten Wirtschaftssektoren geworden. Angesichts einer fortschreitenden europäischen Integration können gerade die Regionen an der Peripherie der einzelnen Staaten mit erheblichen wirtschaftlichen Gunstfaktoren aufwarten. Die Randlage bietet dem internationalen Transportgewerbe, dem Fremdenverkehr, dem Einkaufstourismus bzw. jeglicher Art der grenzüberschreitenden Kooperation günstige Standorte. Nachteile, die in der Phase der Industrialisierung Gültigkeit besaßen, schwächen sich damit allmählich ab. Randgebiete mit Minderheiten können folglich nicht nur in kultureller,sondern auch in wirtschaftlicher Hinsicht Brückenfunktionen übernehmen (ZuPanCIC 1993, Steinicke \& Zupancic 1995). Durch den Fall des Eisernen Vorhangs ist die ehemalige Grenze zum Ostblock zu einem nicht unbedeutenden wirtschaftlichen Impulsgeber geworden, der nicht zuletzt auch den nationalen Minderheiten in Österreich zugute kommen kann (vgl. z.B. STABEL 2001).

Die in den vergangenen Jahrzehnten verstärkte Nachfrage nach städtischen Bildungsmöglichkeiten bzw. die Arbeitsplatzsuche haben dazu geführt, dass zahlreiche Angehörige dieser Minderheiten in die Städte ihres ethnischen Territoriums zuwanderten und nunmehr Teile der (sub)urbanen Bevölkerung geworden sind. Wie Beobachtungen zeigen, ist heute der Wechsel von der ruralen in die urbane Umgebung nicht mehr gleichbedeutend mit einem Abstreifen der alten ethnischen Identität und einer Assimilation an die Mehrheit. Im Gegenteil, es sind gerade die Stadtbewohner, die ihre ethnolinguistische Herkunft bzw. ihre ethnischen Wurzeln neu entdecken, und in den städtischen Zentren bieten sich geeignete Möglichkeiten, die damit in Zusammenhang stehenden kulturellen Ausdrucksformen neu zu beleben. Als Prototyp dafür mag die lateinamerikanischen Zuwanderung in die Vereinigten Staaten gelten, die - ungeachtet jeglichen Melting PotVerständnisses - von den Metropolen aus die «Hispanisierung» des U.S. Südens und Südwestens vorantreibt (Thieme \& Laux 1996, Allen \& Turner 1997).

Aber auch innerhalb der EU machen sich ähnliche Tendenzen bemerkbar. Hier sind es Vertreter von bodenständigen Minderheiten, die von den infrastrukturellen Gegebenheiten urbaner Räume begünstigt ihre mitgebrachte Kultur verstärkt pflegen können. Diese in der Regel gut ausgebildeten städtischen Akteure wirken vielfach als "opinion leaders" und bilden für ihr traditionelles Siedlungsgebiet letztendlich «Multiplikatoren» des Rückbesinnungsgedankens. Dies mindert auch den Schaden, den sie ihrer Her- kunftsregion mit der Abwanderung («brain-drain») zufügten. Beispielsweise ist die in den vergangenen zwei Jahrzehnten durch die Landflucht stark angewachsene nordkorsische Agglomeration Bastia (gemeinsam mit Corte) Ausstrahlungspunkt der traditionellen Inselkultur geworden (CASTELLANI 1994, GaSSER 2001, Meistersheim 2001). Eine ähnliche Rolle hat San Sebastián im spanischen Baskenland übernommen, wo die baskische Kultur (aber auch die ethnopolitischen Bewegungen) trotz des hohen kastilischen und galicischen Bevölkerungsanteils expandiert (KremNITZ 1991, VALANDro 2001). In abgeschwächtem Maße gilt das ebenso für die Sorben in Deutschland, die von den innovativen kulturellen Tätigkeiten in der Stadt Bautzen profitieren (Elle 1992, Stiftung für Das SorbiSCHE VolK 1997, ARBEITSKREIS DER LANDESKUNDLICHEN INSTITUTE \& Sorbisches Institut 1999). Bezeichnenderweise nimmt auch der Anteil von bodenständigen ethnischen Minderheiten in Städten nicht mehr ab: So verdoppelte sich in der Dekade 1981/91 die Zahl der seit über 100 Jahren in Wien ansässigen tschechischen und slowakischen Minorität, also der österreichischen Staatsbürger mit tschechischer oder slowakischer Umgangssprache (ÖSTAT 1983, 1993, ÖSTERREICHISCHES VolKSGRUPPENZENTRUM 1996). Außerdem erwachen gegenwärtig in Wien bislang nicht registrierte städtische Volksgruppen, wie die Wiener Polen oder die Wiener Rumänen (ÖSTERREICHISCHES VOLKSGRUPPENZENTRUM 1997, 2000). Von der ethnischen Renaissance der Kärntner Slowenen soll im folgenden Kapitel mehr die Rede sein. Dabei lassen sich auch die Hauptgründe aufzeigen, warum Städte in zunehmendem Maße die Rolle als Innovationszentrum für eine Neubesinnung ethnischer Minderheiten übernehmen.

\section{Das Beispiel der Kärntner Slowenen}

\subsection{Territorial- und Bevölkerungsverluste}

Das gemischtsprachige Gebiet Kärntens, das den Rest eines einst viel weiter nach Westen und Norden reichenden slowenischen (oder alpenslawischen) Territoriums bildet, erstreckt sich über eine Fläche von rund $2.500 \mathrm{~km}^{2}$ und umfasste im Volkszählungsjahr 1991 - einschließlich der Städte Klagenfurt (89.415 Einw.) und Villach (54.640 Einw.) - eine Wohnbevölkerung von 264.620 Personen. In diesem Raum ergaben sich in den letzten zwei Jahrhunderten zwischen den Deutsch- und Slowenischsprachigen fast alle Formen des Zusammenlebens: Das Spektrum reicht von friedlicher Koexistenz über wirtschaftliche und politische Konflikte bis hin zur offenen Feindschaft, wie es nach dem Ersten Weltkrieg der Fall war. Nach der Volksabstimmung, bei der sich im Jahr 1920 die Mehrheit der Südkärntner für den Verbleib des gemischtsprachigen Gebiets bei Österreich ausgesprochen hatte, folgte eine Zeit, in der die Kärntner 
Slowenen einer besonders aggressiven Assimilierungspolitik ausgesetzt waren. Sie gipfelte darin, dass während des Zweiten Weltkriegs Teile der slowenischen Bevölkerung systematisch ausgesiedelt und verschleppt wurden. Die assimilationsgünstige Situation blieb auch in der Zeit der Zweiten Republik weiterbestehen. Anlässlich des «Ortstafelsturms» wurden noch im Jahr 1972 von beiden Seiten wieder die alten Feindbilder ausgegraben. Im Laufe der achtziger Jahre mit zunehmendem Generationswechsel - entspannte sich jedoch das Verhältnis zwischen den Deutschund Slowenischsprachigen weitgehend. Ein wichtiger Grund dafür waren die sich abzeichnenden Demokratisierungstendenzen in Slowenien. Deutschnationale Kreise verloren damit wichtige Argumentationshilfen in ihrer Propaganda gegen die Kärntner Slowenen (ÖSTERreichisches VolKSGRUPPENZENTRUM 1993).

Der Bevölkerungs- und Raumverlust dauert, wie erwähnt, schon seit Jahrhunderten an. Vertraut man den offiziellen statistischen Daten, so schrumpfte die slowenische Minderheit in Kärnten innerhalb eines Jahrhunderts von 75.136 Personen im Jahr $1900(=21,9 \%$ der Bevölkerung) auf 16.552 im Jahr 1981 bzw. 14.850 im Jahr 1991 (= 2,8\%). Dieser territoriale Rückzug der Kärntner Slowenen ist bereits mehrfach kartographisch veranschaulicht worden (GAMERITH 1993, 1994, Steinicke 1995: 56, Steinicke \& Zupancic 1995: 193).

Der slowenische Anteil umfasste im Volkszählungsjahr 1991 laut amtlichen Quellen 2,8\% der Einwohner Kärntens und 5,6\% des gemischtsprachigen Gebiets. Von den 38 Gemeinden dieser Region wiesen nur Zell (93,5\%) und Globasnitz (51,6\%) eine slowenische Mehrheit auf.

Es gilt jedoch als gesichert, dass die Zensusdaten nicht mit den tatsächlichen Sprachverhältnissen übereinstimmen. Folgende Hauptgründe sind dafür anzuführen: Obwohl erst im Zensus von 1991 die nicht eindeutig festlegbare «Umgangssprache» in Richtung «Familiensprache» (i.S. des Bekenntnisprinzips) definiert wurde, bestehen weiterhin gravierende Inkonsequenzen der österreichischen Sprachenzählung, die sich zum Teil bis in die nationalsozialistische Zeit zurückführen lassen und noch in der Gegenwart beibehalten werden (vgl. Tab. 1): Mit der Einführung der statistischen Kategorie "Windische» (= deutsch gesinnte Sprachslowenen; vgl. UNKART et al. 1984: 41-43) im Zensus von 1939 teilte man die Kärntner Slowenen in zwei Gruppen, was sich in ethnopsychologischer Hinsicht bis heute ungünstig auf die Einheit der Volksgruppe auswirkt. Überdies ist auch die Einführung von weiteren statistischen Sprachkategorien im Jahr 1951 als eine Spaltung der Minderheit zu verstehen (z. B. die Sprachkombinationen DeutschSlowenisch, Slowenisch-Deutsch, Deutsch-Windisch usw.).

Ein letzter Hauptgrund ist ebenso im psychologischen Bereich angesiedelt. Besonders in den westlichen und nördlichen Randzonen des gemischtsprachigen Gebiets sind bei einem Gutteil der Kärntner Slowenen Tendenzen der ethnischen Selbstentfremdung bezeichnend. Das jahrhundertelange Zusammensiedeln mit der Mehrheitsbevölkerung, bei dem die Slowenen die untere Sozialkategorie bildeten, bewirkte vielfach ein Bekenntnis zur Deutschkärntner Kultur. Soziale Aufstiegschancen wurden damit erleichtert.

Das Ergebnis der amtlichen Sprachenerhebung ist daher sehr oft Gegenstand von wissenschaftlicher Kritik gewesen, auf die hier allerdings nicht eingegangen werden soll (vgl. z. B. KLEMENCIC 1977, REITERER

\begin{tabular}{|c|c|c|}
\hline ZENSUSJAHR & SLOWENEN & WINDISCHE \\
\hline 1939 & 21.701 & 21.478 \\
1951 & 22.367 & 19.728 \\
1961 & 14.001 & 11.469 \\
1971 & 17.014 & 3.914 \\
1981 & 14.204 & 2.348 \\
1991 & 13.962 & 888 \\
\hline
\end{tabular}

Tab. 1: Die Zahl der Kärntner Slowenen und Windischen in der amtlichen Sprachenzählung

The number of Slovenes and "Windische" according to the official census

Le nombre de Slovènes de Carinthie et de "Windische» dans le recensement officiel des langues

Quelle: IBouNig 1986, ÖSTAT 1993 
1986, Grafenauer 1990, Zupancic 1992, 1999). Das zahlenmäßige Kräfteverhältnis der Volksgruppen in Kärnten würde anders aussehen, wenn man sich nicht auf die offizielle Statistik, sondern auf gut fundierte Schätzungen stützte. Beispielsweise legte ZuPANCIC $(1992,1993)$ auf Grundlage der Schul-, Medien- und Kirchenstatistik, der Auswertung von slowenischen Grabinschriften sowie der Berücksichtigung anderer Expertenschätzungen (z.B. REITERER 1986) die Zahl der Slowenischsprachigen in Kärnten («Sprachslowenen») mit rund 50.000 Personen fest. Sie ist damit mehr als dreimal so hoch wie die amtliche österreichische Sprachenerhebung sie ausweist.

\subsection{Eine Trendwende in Südkärnten}

Die slowenische Minderheit in Kärnten genießt durch den Staatsvertrag von 1955 einen international abgesicherten Schutz, der durch das Volksgruppengesetz von 1976 konkretisiert wurde. Die Gewährung der damit verbundenen besonderen Rechte im Bereich der Amtssprache (inkl. topographischen Bezeichnungen), im Erziehungs- und Schulwesen sowie in der medialen Versorgung ist allerdings weitgehend vom zahlenmäßigen Kräfteverhältnis zwischen Minderheit und Mehrheit in den jeweiligen Gemeinden des gemischtsprachigen Teils Kärntens abhängig. Die slowenischen Gruppenorganisationen sind mit dieser amtlichen Rechtsauffassung nicht einverstanden, da durch den Zensus nicht die tatsächlichen sprachlichen Verhältnisse festgestellt werden (vgl.ÖSTERREICHISCHES VOLKSGRUPPENZENTRUM 1996: 19).

Mag es auch nach den bisherigen Ausführungen über die ethnopolitische Situation bei den Kärntner Slowenen überraschen, so kündigt sich in Südkärnten seit den achtziger Jahren eine Abschwächung des Assimilationsprozesses an. Einen ersten Hinweis dafür liefern die Zensusergebnisse von 1991. Es lässt sich feststellen, dass der Rückgang der Zahl der Slowenen seit 1981 deutlich geringer war als in den vorhergehenden Dekaden. Zwar verlor die Minderheit weitere 1.702 Personen $(=-10,3 \%)$, v.a. in den «slowenischen Kerngebieten» im Südosten des Landes (z.B. Bleiburg, Globasnitz oder Eisenkappel-Vellach), doch in nicht weniger als zwölf von 38 Gemeinden des gemischtsprachigen Gebiets nahm ihre Zahl erstmals wieder zu (vgl. Abb. 2).

Dieser Zuwachs spielt sich einerseits an der Peripherie des gemischtsprachigen Territoriums ab, wo

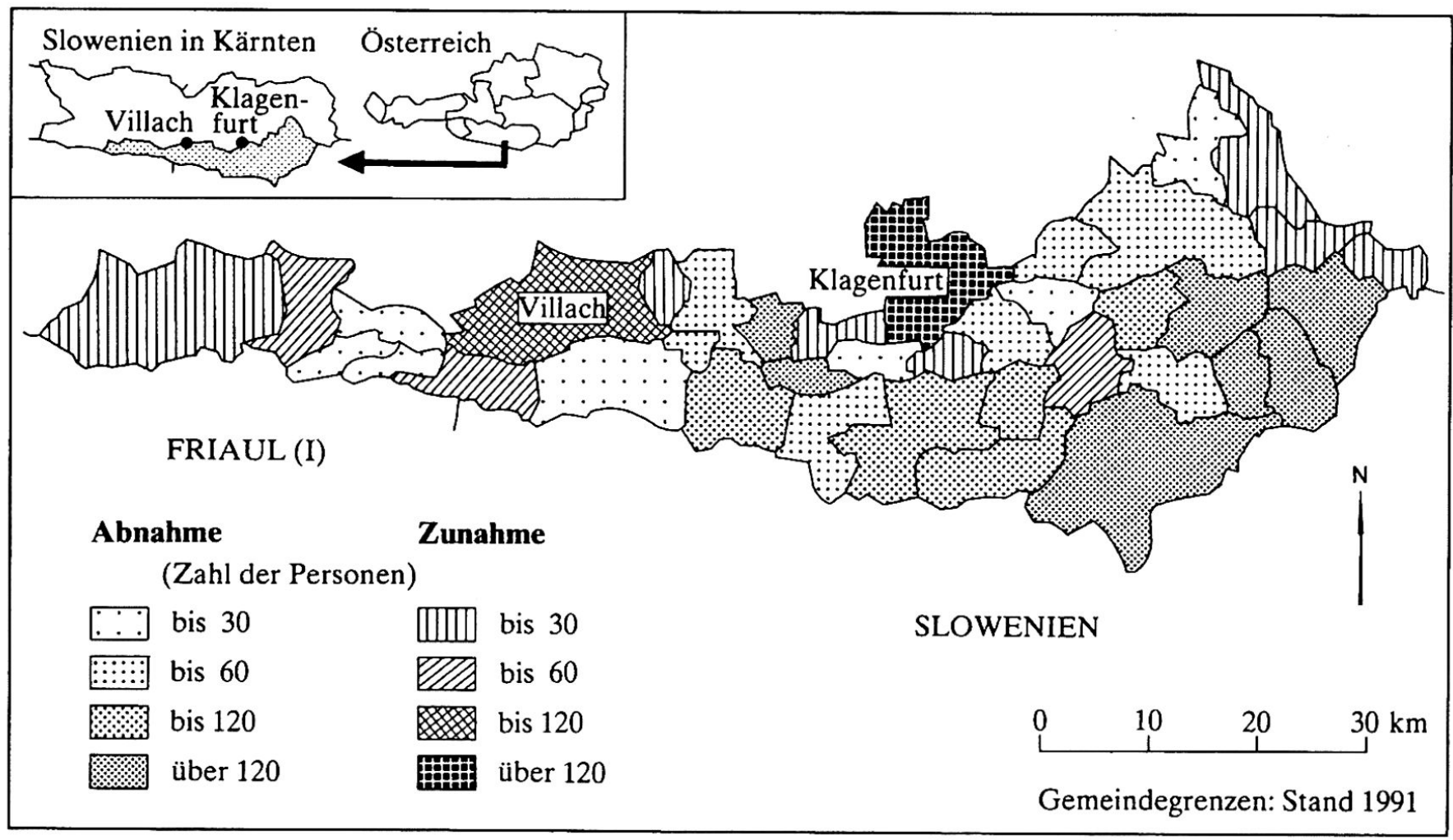

Abb. 2: Die Entwicklung der Zahl der slowenischsprachigen Inländer Südkärntens 1981 und 1991

Development of Slovene population in Southern Carinthia 1981-1991 (Austrian citizens)

L'évolution de la population de langue slovène en Carinthie du Sud, entre 1981 et 1991 (citoyens autrichiens)

Quelle: ÖSTAT 1983, 1993; Entwurf: E. STEINICKE, Bearbeitung: L. BAUMANN 
Änderungen im ethnischen Bekenntnis dafür ausschlaggebend sind. Immer mehr «Sprachslowenen», die sich bislang als Deutschkärntner bekannten, finden demnach zur slowenischen Identität zurück. Um den spekulativen Anstrich dieser Erklärung zu verringern, wurden in den Untergailtaler Gemeinden Hermagor und St. Stefan Befragungen durchgeführt. Die Ergebnisse unterstrichen die These der ethnischen Umorientierung (Steinicke 1995). Zum anderen ist es bezeichnend, dass erstmals in den beiden Städten Klagenfurt $(+28,1 \%)$ und Villach $(+70,5 \%)$ sowie in verschiedenen Suburbia-Gemeinden ein Wachsen der Minderheit feststellbar war. Der Großteil des zahlenmäßigen Anstiegs hängt mit innerregionalen Wanderungen der slowenischen Minorität zusammen. Gegenüber den vergangenen Dekaden fällt aber auf, dass dieser Personenkreis die ethnische Herkunft nicht mehr verleugnet und sich offen zur slowenischen Ethnie bekennt. Der restliche Teil des Zuwachses ist - wie in den angesprochenen Periphergebieten - wiederum nur durch eine ethnische Rückbesinnung erklärbar. Dies bestätigt außerdem der rapide zahlenmäßige Rückgang der «Windischen» (vgl.Tab.1). Schon im Volkszählungsjahr 1971 hatten sich kaum noch Personen mit höherer Schulbildung als «Windische» deklariert (MeusBurGER 1979: 261).

Noch geringer wären die Verluste der Kärntner Slowenen seit 1981, wenn man die gesamte Wohnbevölkerung als Berechnungsgrundlage nimmt, also auch die in Kärnten wohnhaften Ausländer berücksichtigt. Demnach beträgt die Abnahme seit 1981 für das Land nur $3,7 \%$, in Klagenfurt $(+46 \%)$ und Villach $(+177,9 \%)$ sind sogar enorme Zuwächse im Anteil der Slowenen zu verzeichnen. Es ist naheliegend, daraus einen Zuzug aus Slowenien zu folgern.

Die Studien von Gamerith $(1993,1994)$ ergaben, dass von der immer wieder vorgebrachten Feststellung einer wirtschaftlichen Benachteiligung der Minderheit Südkärntens in landwirtschaftlicher, gewerblich-industrieller und sozialer Hinsicht heute keine Rede mehr sein kann, wenn auch noch im Jahr 1981 zehn Gemeinden des gemischtsprachigen Teils zu den strukturschwachen Gebieten Kärntens zählten. Aber auch sie gehörten schon damals nicht zu den ausgesprochenen Passivräumen Kärntens. Gewisse Merkmale, wie die Jugendquote, die Wanderungsbilanz oder der Anteil an Studenten und Akademikern, fielen dort sogar günstig aus. Insgesamt stieg im gemischtsprachigen Teil Kärntens die Wohnbevölkerung zwischen 1951 und 1991 um ein Viertel.

War die slowenische Minderheit im Jahr 1970 noch vorwiegend agrarisch orientiert, so änderte sich in der Folge die Sozialstruktur stärker als anderswo. In seinen Studien über das Ausbildungsniveau der ethnischen
Minderheiten in Österreich für das Jahr 1971 sprach Meusburger (1979:258f., 1980:147f.) den sich abzeichnenden sozialen Aufschwung der Kärntner Slowenen an. Dabei wies er auf die im internationalen Vergleich günstige Schulsituation im gemischtsprachigen Teil Kärntens hin und hob besonders das hohe Qualifikationsniveau der aus der Minderheit stammenden Lehrpersonen für den Slowenischunterricht hervor. Bestand im Jahr 1971 bereits eine nennenswerte Zahl an mittleren und hohen Beamtenstellen, die von Kärntner Slowenen bekleidet wurden, so stieg in den darauf folgenden Jahren der Akademikeranteil innerhalb der slowenischen Volksgruppe des gemischtsprachigen Territoriums überdurchschnittlich an. Im Jahr 1981 fiel im Vergleich der slowenisch- mit der deutschsprachigen Bevölkerung bereits die deutlich höhere Akademikerquote der Slowenen auf (2,1 bzw. 1,5\%). Die Tabellen in der Studie von Iвounig (1986: 49-51 und 90-93) machen diesen bildungsmäßigen Aufholprozess der Slowenen deutlich, der im Jahr 1981 bereits in einem «Überholen» der deutschsprachigen Bevölkerung unter 30 Jahren mündete. Heute stellen die Kärntner Slowenen in ihrem Siedlungsgebiet nicht nur einen Gutteil der Unternehmerschaft, sie liegen nach den empirischen Untersuchungen von ZuPANCIC (1992: 210-272, 1999: 122-139 und 227f.) im Bildungsund Qualifikationsniveau auch eindeutig über dem Kärntner Schnitt.

Der zuletzt genannte Aspekt ist zweifellos mit dem Einfluss des slowenischen Gymnasiums in Klagenfurt, das 1957 gegründet wurde, verbunden. Es bietet die Möglichkeit, neben einer zweisprachig vermittelten Allgemeinbildung die Kultur und Geschichte der Slowenen kennen zu lernen, was das ethnische Selbstverständnis stärkte. Wie aus den Untersuchungen von Zupancic $(1992,1993)$ hervorgeht, besuchen im Schnitt ca. 440 Jugendliche, rund 90\% davon Südkärntner, dieses Gymnasium. Eine Hochrechnung des ÖSTERREICHISCHEN VOLKSGRUPPENZENTRUMS (2000) ergab, dass seit dem Bestehen des Slowenischen Gymnasiums mehr als ein Drittel aller Kärntner Slowenen, die sich auch als solche bezeichnen, zumindest ein Schuljahr lang diese Schule besucht haben. Die Gesamtzahl der Absolventen beträgt seit 1957 knapp 4000. Wie Meusburger im Jahr 1979 erahnte, ist aus ihrem Kreis eine intellektuelle Schicht entstanden, welche die führende Rolle der Minderheit übernommen hat (ReITERER 1986, ZuPANCIC 1992, 1999). Es ist zu erwarten, dass sich dies aufgrund der vor einigen Jahren in Klagenfurt errichteten zweisprachigen Handelsakademie demnächst verstärken wird. Mit dem Anheben des Bildungsniveaus machte sich gleichzeitig eine Zuwanderung der slowenischen Minderheit in die zentralen Orte Kärntens - vor allem nach Klagenfurt und Villach - aber auch in die Bundeshauptstadt Wien bemerkbar (ZuPANCIC 1999). In 
den genannten Städten sind Kärntner Slowenen schon seit den achtziger Jahren in höheren Berufen (Professoren, Verleger, Ärzte, Juristen, Lehrer) vertreten. Sie bilden die Akteure, die sowohl über persönlichen Kontakt als auch über die zwei großen slowenischen Organisationen in Kärnten die Innovation der ethnischen Rückbesinnung in die Peripherie tragen. Inzwischen haben Kärntner Slowenen auch leitende Funktionen im Österreichischen Volksgruppenzentrum in Wien, in verschiedenen Verlagen sowie in den Minderheitenredaktionen des ORF (Wien und Klagenfurt) inne. Sie sind praktisch zu den Sprechern der ethnolinguistischen Minoritäten Österreichs geworden.

Insgesamt zeigt die neue Entwicklung, dass auch der ländliche Raum Südkärntens in die Phase der Tertiärisierung eingetreten ist. Dabei konnte die slowenische Sprache an Prestige gewinnen. Dies hängt im wesentlichen mit dem Entstehen einer breiten gebildeten und inzwischen urban geprägten Schicht bei den Kärntner Slowenen zusammen, der es immer mehr gelingt, kulturpolitische Forderungen durchzusetzen. Sie hatte auch maßgeblichen Einfluss auf ökonomische Verbesserungen im gemischtsprachigen Territorium. Über solche gruppenverstärkende Faktoren, wie der Bedeutungsgewinn Südkärntens im Tourismus, die wirtschaftliche Verbindung mit Slowenien oder die slowenischen Betriebsansiedelungen bzw. Zuwanderungen aus Slowenien, wurde bereits an anderer Stelle ausführlich berichtet (STEINICKE 1995, 2001b).

\section{Fazit: Städtische Akteure und ethnische Rückbesinnung}

Das Beispiel der Kärntner Slowenen gibt einen neuen Trend zu erkennen, dessen Intensität sich mit der Auswertung des Zensus 2001 auch zahlenmäßig umreißen lassen wird: Wie angeführt, leben in Kärnten ca. 50.000 Sprachslowenen, von denen sich lediglich ein knappes Drittel zur slowenischen Minderheit bekennt. Insofern wäre es durchaus vorstellbar, dass in Südkärnten ein Umdenken einsetzt und große Teile der zweisprachigen Bewohner, die bisher mehr der Deutschkärntner Kultur nahe standen, sich wieder ihrer ethnischen Herkunft besinnen.

Voraussetzung für eine ähnliche Entwicklung in anderen Räumen ist die staatliche Anerkennung der jeweiligen Minorität, mit der in der Regel ein Schutzmechanismus verbunden ist. Dieser kann sich auf bloße kulturelle Ausnahmeregelungen beschränken, er kann aber auch politische Mitbestimmung der Minderheit vorsehen und bis hin zu territorial autonomen Regelungen (Autonomie der Minderheit) reichen. Beispielsweise lässt sich der amtlich geregelte Schutz der Kärntner Slowenen im EU-weiten Vergleich als «gut» einstufen, selbst wenn er nicht jene Standards erreicht, die in Südtirol, im Aostatal oder in Katalonien Anwendung finden.

Obwohl noch immer hinreichende Minderheitenregelungen auf rechtlich-demokratischer Grundlage fehlen, hat sich in den meisten Ländern des westlichen Europa die Ansicht durchgesetzt, Minderheiten das Recht auf Gruppenschutz sowie gewisse Rechte auf besonderen Schutz (z.B. das Recht auf eigene Sprache und Schulen oder auf eigene Organisationen) zuzuerkennen. Kommen diese Schutzgedanken zur Umsetzung, so entstehen als Konsequenz zentrale Einrichtungen, die den ethnischen Minderheiten zugute kommen sollen. Die Standortwahl für eine solche Infrastruktur folgt in der Regel der Logik des Zentrale-Orte-Systems. Städte in Minderheitengebieten werden dadurch Mittelpunkte für Institutionen, in denen die kulturellen Traditionen der Minderheiten gepflegt und vermittelt werden. In erster Linie betrifft dies die Bildungseinrichtungen. Gelingt es, in den Schulen (Universitäten) das ethnische Selbstverständnis bei den jungen Minderheitenangehörigen zu wecken, so ist das Beispiel der Kärntner Slowenen durchaus auch auf andere Minderheitengebiete übertragbar: Wichtig ist es festzuhalten, dass diese gut ausgebildeten, sich ihrer ethnischen Sonderstellung bewussten Absolventen von höheren Schulen (sowie Universitäten), egal ob sie im (sub)urbanen Raum verbleiben oder in die ländlichen Gebiete zurückwandern, der Minderheit nicht mehr abhanden kommen. Dieser mehrsprachige und volkstumsbewusste Personenkreis wird sich in der Folge als Hauptakteur für die Diffusion der ethnischen Rückbesinnung in das umliegende Minderheitenterritorium erweisen. Als Resultat ergibt sich zwangsläufig eine allgemeine Stärkung der Minderheit und damit auch ein Bedeutungszuwachs des Minderheitenterritoriums.

Der Zeitgeist unterstützt eine solche Entwicklung. Die im Zusammenhang mit der Globalisierung zu sehende Auflösung nationalstaatlicher Wirtschaftsräume führt zu Gegenbewegungen, die sich u.a. in der Hervorhebung des Regionalen bemerkbar machen. Das Besondere einer Region, und hier in erster Linie die ethnische Identität als wesentlicher Teil einer regionalen Identität, wird in zunehmendem Maße bedeutend. In diese Richtung wirkt auch das vielpropagierte Europa der Regionen. Kulturelle Singularitäten - wie eben die Ethnodiversität - werden nicht mehr versteckt gehalten, sondern vielfach als Bereicherung im Wettbewerb der Regionen untereinander betrachtet.

Zusammenfassend lässt sich festhalten, dass Städte in Minderheitengebieten keinesfalls nur mehr in eine assimilatorische Richtung hin wirken. Durch Maßnahmen des Minderheitenschutzes sowie durch die Gunstfak- 
toren städtischer Infrastruktur entstehen gerade in den Verdichtungsräumen Tendenzen, die eine Chance für die Bewahrung der ethnischen Vielfalt bieten. Um die These von den Städten als Innovationszentren für eine Neubesinnung ethnischer Minderheiten in den Rang eines theoretischen Ansatzes zu heben, bedarf es zweifellos weiterer empirischer Forschungsergebnisse, welche die zentralen Aussagen der vorliegende Studie absichern und präzisieren. In einem weiteren Schritt ist auch das Konfliktpotenzial zwischen Minderheit und Mehrheit zu analysieren, das sich mit dem hier skizzierten neuen ethnischen Selbstverständnis auftun kann.

\section{Literatur}

Allen, J.P. \& E. Turner (1997): The Ethnic Quilt: Population Diversity in Southern California. - Northridge, CA: The Center for Geographical Studies, California State University.

ARbeitskreis DeR LANDESKUndLichen INSTITUTE UND ForSCHUNGSSTELLEN IN DER DEUTSCHEN AKADEMIE FÜR LANDESKUNDE \& Sorbisches InStITUT (1999): Tagung «Autochthone ethnische Minderheiten in Mitteleuropa - Entwicklung, Probleme und Schutzziele» vom 13.-15.05.1999. Tagungsunterlagen. - Bautzen.

Barth, F. (ed.) (1969): Ethnic Groups and Boundaries. The Social Organization of Culture Difference. - Bergen, Oslo: Universitetsforlaget, London: George Allen and Unwin.

Breton, R. (1983): Lob der Verschiedenheit. - = Ethnos 25, Wien.

Castellani, M. (1994): Une montagne dans l'amer. Corse économique et humaine du passé et d'aujourd'hui. - Genova: Brigati Glauco.

CBS (Centraal Bureau voor de Statistiek), Divisie IV, Sector Bevolking (1999): Niet-Nederlanders in Nederland. - Voorburg-Heerlen.

ElLe, L. (1992): Zur aktuellen Sprachsituation der Lausitzer Sorben. - In: Europa Ethnica 49: 1-12.

EnTZinger, H. (1998): Zu einem Modell der Inkorporation von Einwanderern: das Beispiel der Niederlande. - In: Bommes, M. \& J. Halfmann (Hrsg.): Migration in nationalen Wohlfahrtsstaaten. Theoretische und vergleichende Untersuchungen. - Osnabrück: Universitäts-Verlag Rasch: 105-122.

ERIKSEN, K. et al. (1991): Governments and the Education of Non-Dominant Ethnic Groups in Comparative Perspective. - In: TomiaK, J. et al. (eds): Schooling, Educational Policy and Ethnic Identity. Comparative Studies on Governments and Non-dominant Ethnic Groups in Europe, 1850-1940. - Aldershot: Dartmouth Publishing, New York: New York University Press: 389-417.

FassmanN, H. \& R. Münz (eds) (1994): European Migration in the Late Twentieth Century. Historical Patterns, Actual Trends, and Social Implications. Hants, GB: EE-Publ.
Fenet, A. et al. (1995): Le Droit et les Minorités. Analyses et Textes. - Bruxelles: Bruylant.

GAMERITH, W. (1993): Geographische Untersuchungen über die Kärntner Slowenen als ethnische Minderheit in peripherer Lage. - Dissertation am Geographischen Institut der Universität Salzburg.

Gamerith, W. (1994): Ethnizität und ihr zeitlichräumlicher Wandel. Das Beispiel der Kärntner Slowenen. - = Klagenfurter Geographische Hefte 12.

GASSER, S. (2001): Korsika. Bevölkerungsdynamischer Umbruch in der Gegenwart. - Unveröffentlichte Diplomarbeit am Institut für Geographie der Universität Innsbruck.

Glebe, G. \& J. O'Loughlin (eds) (1987): Foreign Minorities in Continental European Cities. - = Erdkundliches Wissen 84. - Stuttgart: Steiner

Grafenauer, B. (1990): Poucevanje problematike posameznih manjsinskih skupnosti. Narodne manjsine. Ljubljana: SAZU.

HaArmann, H. (1983): Elemente einer Soziologie der kleinen Sprachen Europas. - Bd. 1: Materialien zur Sprachökologie, 3. Auflage, Hamburg: Dtv.

Heckmann, F. (1978): Minderheiten. Begriffsanalyse und Entwicklung einer historisch-systematischen Typologie. - In: Kölner Zeitschrift für Soziologie und Sozialpsychologie 30: 761-779.

Heckmann, F. (1992): Ethnische Minderheiten, Volk und Nation. Soziologie inter-ethnischer Beziehungen. - Stuttgart: Ferdinand Enke Verlag.

Héraud, G. (1993): L'Europe des Ethnies. - Bruxelles, Paris: Bruyant.

Iвounig, P. (1986): Die Kärntner Slowenen im Spiegel der Volkszählung 1981. - Kärnten Dokumentation, Bd. 2. - Klagenfurt: Amt der Kärntner Landesregierung. KäUfeler, H. (1994): Annäherung an das Prädikat «ethnisch». - In: MülleR, H.-P. (Hrsg.): Ethnische Dynamik in der aussereuropäischen Welt. - Zürich: Argonaut Verlag: 15-25.

KLEMENCIC, V. (1977): Manipulation mit statistischen Daten zum Nachteil der Minderheiten in Österreich. - In: OsolNIK, B. (Hrsg.): Das Minderheitenproblem in den jugoslawisch-österreichischen Beziehungen. Beograd: Jugoslovenska Stvarnost: 143-162.

KLoss, H. (1969): Grundfragen der Ethnopolitik im 20. Jahrhundert. $-=$ Ethnos 7, Wien: Braumüller.

Koter, M. (1993): Geographical Classification of Ethnic Minorities. - In: Geographica Slovenica 24 (Geography and Ethnicity): 123-138.

KRAAS-SCHNEIDER F. (1989): Bevölkerungsgruppen und Minoritäten. Handbuch der ethnischen, sprachlichen und religiösen Bevölkerungsgruppen der Welt. - Stuttgart, Wiesbaden: Steiner.

KraAs, F. (1992a): Die Rätoromanen Graubündens. Stuttgart: Steiner.

KraAs, F. (1992b): Ethnolinguistische Bevölkerungsgruppen und Minoritäten als Aufgabe geographischer Forschung. - In: Die Erde 123: 177-190. 
Kremnitz, G. (1991): Aktuelle Probleme der Sprachpolitik in Euskadi. - In: Europa Ethnica 48: 10-23.

Meistersheim, A. (ed.) (1999): L'Ile laboratoire. Ajaccio: Piazzola.

Meistersheim, A. (2001): Figures de l'île. - Ajaccio: DCL.

Meusburger, P. (1979): Ausbildungsniveau und schulische Situation der Slowenen in Kärnten von 1848 bis 1978. - In: Innsbrucker Geographische Studien 5: 229-264.

Meusburger, P. (1980): Beiträge zur Geographie des Bildungs- und Qualifikationswesens. - = Innsbrucker Geographische Studien 7.

Meusburger, P. (1996): Educational Achievement, Language of Instruction, and School System as Key Elements of Minority Research. - In: Frantz, K. \& A. Sauder (eds): Ethnic Persistence and Change in Europe and America. Traces in Landscape and Society. - University of Innsbruck: 187-222.

Meusburger, P. (1998): Bildungsgeographie - Wissen und Ausbildung in der räumlichen Dimension. - Heidelberg, Berlin: Akad. Verlag.

MühlmanN, W.E. (1964): Rassen, Ethnien, Kulturen. Neuwied u.a.: Luchterhand.

Nelde, P.H. (1987): Langues en contact et en conflit. In: Europa Ethnica 44: 63-72.

ÖSTERREICHISCHES VolKSGRUPPENZENTRUM (1993): Kärntner Slowenen. - Band 1, 2., aktualisierte Auflage, Wien: Österreichische Volksgruppenhandbücher.

ÖSTERREICHISCHES VOLKSGRUPPENZENTRUM (1996): Volksgruppenreport 1996. - Wien.

ÖSTERREICHISCHES VOLKSGRUPPENZENTRUM (1997): 2. Oberwarter Volksgruppenkongress, 22. Feb. 1997, Tagungsunterlagen. - Oberwart.

ÖSTERREICHISCHES VOLKSGRUPPENZENTRUM (2000): Bericht zur Durchführung des Europäischen Rahmenübereinkommens zum Schutz nationaler Minderheiten in der Republik Österreich. - Unveröffentlichter NGOReport, Wien.

ÖSTAT, Österreichisches Statistisches ZentralAMT (1983): Volkszählung 1981, Hauptergebnisse I, Kärnten. - Wien.

ÖSTAT, Österreichisches Statistisches ZentralAMT (1993): Volkszählung 1991, Hauptergebnisse I, Kärnten. - Wien.

Pan, C. \& B.S. Pfell (2000): Die Volksgruppen in Europa. Ein Handbuch. - = Ethnos 56, Wien.

Plasseraud, Y. (1998): Les minorités. - Paris: Montchrestien.

Pletsch, A. (ed.) (1985): Ethnicity in Canada. International Examples and Perspectives. $-=$ Marburger Geographische Schriften 96.

ReITERER, A. F. (1986): Doktor und Bauer. Ethnischer Konflikt und sozialer Wandel: Die Sozialstruktur der slowenischen Minderheit in Kärnten. - Klagenfurt: Drava.

SANGUin, A.-L. (1993): Quelles minorités pour quels territoires? - In: SANGUin A.-L. (ed.): Les minorités ethniques en Europe. - Paris: Ed. L'Harmattan: 5-18.

Stabel, B. (2001): Die Burgenlandkroaten - zwischen Assimilation und ethnischer Renaissance. - Unveröffentlichte Diplomarbeit am Institut für Geographie der Universität Innsbruck.

SteinicKe, E. (1991): Friaul - Bevölkerung und Ethnizität. - = Innsbrucker Geographische Studien 20.

Steinicke, E. (1995): Die Slowenen in Kärnten und Friaul - eine verschwindende Minderheit. - In: Geographische Rundschau 47: 52-57.

STEINICKE, E. (2001a): «Ethnische Minderheiten» ein Begriffsverständnis in globaler Perspektive. In: Heller, H. (Hrsg.): Neue Heimat Deutschland. Aspekte der Zuwanderung, Akkulturation und emotionalen Bindung. - = Erlanger Forschungen Reihe A-Geisteswissenschaften 95 (im Druck).

Steinicke, E. (2001b): Die Kärntner Slowenen und die Südtiroler Ladiner. Eine Renaissance kleiner ethnischer Minderheiten? - In: Berichte zur deutschen Landeskunde 75 (im Druck).

Steinicke, E. \& J. Zupancic (1995): Les slovènes de Carinthie, une communauté de la périphérie? - In: Goetschy, H. \& A.-L. Sanguin (ed.): Langues régionales et relations transfrontalières en Europe. - Paris: Ed. L'Harmattan: 189-203.

StifTUNG FÜr DAS SORBISCHE VOLK (Hrsg.) (1997): Serbja - Die Sorben in Deutschland. - Görlitz: Maxroi.

Thieme, G. \& H.D. Laux (1996): Los Angeles - A Multi-Ethnic Metropolis Special Patterns and SocioEconomic Problem as a Result of Recent Migration Processes. - In: Frantz, K. (ed.): Human Geography in North America, New Perspectives and Trends in Research. - = Innsbrucker Geographische Studien 26: 81-96.

TomiaK, J. \& A. Kazamias (1991): Introduction. - In: TomiaK, J. et al. (ed.): Schooling, Educational Policy and Ethnic Identity. Comparative Studies on Governments and Non-dominant Ethnic Groups in Europe, 1850-1940. - Aldershot: Dartmouth Publishing, New York: New York University Press: 1-11.

UNKART, R. et al. (1984): Zur Lage der Slowenen in Kärnten. Die slowenische Volksgruppe und die Wahlkreiseinteilung 1979 - eine Dokumentation. - Klagenfurt: Kärntner Landesarchiv.

U.S. DePartment of Commerce, Bureau of the Census: Censuses of 1900-1990. - Washington D.C.

VALANDRo, F. (2001): Das Baskenland und Nordirland. Eine vergleichende Konfliktanalyse. - Innsbruck u.a.: Studienverlag.

Veiter, T. (1984): Nationalitätenkonflikt und Volksgruppenrecht im ausgehenden 20. Jahrhundert. - Bd. 1,2. Auflage, München: Bayer. Landeszentrale für politische Bildungsarbeit.

WARREN, R. (1982): Schooling, Biculturalism, and Ethnic Identity: A Case Study. - In: SPINDleR, G. (ed.): 
Doing the Ethnography of Schooling: Educational Anthropology for Action. - New York, London: Holt, Rinehart and Winston: 382-409.

WerLen, B. (2000): Sozialgeographie. Eine Einführung. - Bern u.a.: UTB.

ZUPANCIC, J. (1992): Vpliv socialnogeografske preobrazbe na polozaj slovenske manjsine na avstrijskem Koroskem. - Magistrska naloga, univerza v Ljubljani, filozofska fakulteta, Ljubljana.

Zupancic, J. (1993): Socialgeographic Transformation and National Identity - the Case of the Slovene Minority in Carinthia (Austria). - In: GeoJournal 30: 231-234.

Zupancic, J. (1999): Slovenci v Avstriji. - = Geographica Slovenica 32, Ljubljana: 1-245.

\section{Zusammenfassung: Städte als Innovationszentren für eine Neubesinnung ethnischer Minderheiten. Das Beispiel der Kärntner Slowenen (Österreich)}

Vorliegende Studie geht von der These aus, dass urbane Räume in der postindustriellen Phase günstige Handlungsstrukturen bieten, um den Erhalt autochthoner ethnolinguistischer Minderheiten zu fördern. Das Beispiel der slowenischen Minderheit in Kärnten soll diese Annahme veranschaulichen und erklären. Die Zensusergebnisse von 1991 weisen in Südkärnten erstmals auf eine Abschwächung des Assimilationsprozesses hin. Besonders auffallend ist der zahlenmäßige Anstieg der slowenischsprachigen Kärntner in den Stadtregionen von Klagenfurt und Villach. Als Grund dafür wird eine ethnische Rückbesinnung gesehen, die unmittelbar mit der Standortwahl der kulturellen Einrichtungen für die Minderheit zusammenhängt. Nach der Logik der zentralörtlichen Hierarchie bietet sich dafür der urbane Raum an. Hervorzuheben ist dabei das Slowenische Gymnasium in Klagenfurt, dessen Absolventen heute die führende Rolle der Minderheit in Südkärnten übernommen haben. Sie bilden die Akteure, welche die Innovation der ethnischen Rückbesinnung auch in die Peripherie tragen. Diese Entwicklung ist durchaus auch auf andere Räume übertragbar - vorausgesetzt es besteht ein Minderheitenschutz. Städte in Minderheitengebieten werden dadurch zu Schaltstellen für die Pflege und Bewahrung der Minderheitenkultur. Unterstützung erfährt diese Tendenz durch eine neue Sichtweise des Regionalen. Immer mehr werden kulturelle Singularitäten, wie die Ethnodiversität, als Bereicherung im Wettbewerb der Regionen gesehen.

Summary: Urbanised Areas as Innovation Centres for the Revival of Ethnic Minorities. The example of the Carinthian Slovenes

The present study builds on the thesis that in the postindustrialised phase urbanised areas offer favourable frameworks for the maintenance of autochthonous ethno-linguistic minorities. The case study of the Carinthian Slovenes should illustrate and explain this basic assumption. The census results of 1991 for the first time show a weakening of the assimilation process in South Carinthia. What is striking is the significant growth of the Slovene minority in urban and suburban areas of Klagenfurt and Villach. The main reason for this development can be found in the changing ethnic identification within the Slovene group. This ethnic revival depends largely on the localisation of the cultural institutions serving the minority. According to the central places theory, urbanised areas are considered appropriate locations for their establishments. In this context, the Slovene Secondary School of Klagenfurt plays an essential role. The emergence of a large, well-educated Slovene-speaking social elite, whose cultural and political demands are increasingly being accepted, can mainly be attributed to the institution mentioned above. This urban group who found its "way back» to a Slovene identity represents the protagonists who spread the ethnic renaissance also to the peripheral zones. It goes without saying that this development is regionally transferable - on the condition that minority protection legislation exists. Thus, urban and suburban zones will become key areas for the maintenance of ethnic minorities. This trend is supported by a new view of the regional factor, too. To a growing extent cultural singularities, e.g. ethnic diversity, are considered an enrichment for one region's strengths in competing with others.

\section{Résumé: Les agglomérations comme centres d'innovation pour une renaissance des minorités ethniques. L'exemple des Slovènes de Carinthie}

La présente étude part de la thèse selon laquelle les espaces urbains offrent durant la phase postindustrielle des conditions favorables à la promotion des minorités linguistiques. La thèse sera illustrée et expliquée par l'exemple des Slovènes de Carinthie. Pour la première fois le recensement officiel du 1991 montre l'affaiblissement du processus d'assimilation en Carinthie du Sud. Le caractère marquant est le fait que les Slovènes ont vu leur nombre augmenter dans les agglomérations de Klagenfurt et Villach. La raison de cet accroissement est due principalement à des changements dans le sentiment d'appartenance ethnique. Cette renaissance est liée en premier lieu à la localisation des institutions culturelles disponible pour les minorités. Suivant la théorie des lieux centraux, leurs emplacements se concentrent dans les espaces urbains. Le Lycée Slovène de Klagenfurt y joue un rôle important. Son influence a conduit à la formation d'une large couche cultivée parmi les Slovènes de Carinthie qui a réussi à prendre la tête des minorités en Carinthie méridionale. Elle représente les acteurs qui propagent la renaissance ethnique jusque dans les zones périphériques. Cette évolution est absolument transféra- 
ble à d'autres territoires - sous réserve qu'il y existe une législation protectrice des minorités. En conséquence, sur les territoires des minorités les espaces urbains jouent un rôle central pour la sauvegarde des cultures minoritaires. Ces tendances sont confortées par le renouveau du fait régional. De plus en plus, des singularités culturelles - c'est à dire la diversité ethnique - sont considérées comme un enrichissement dans la compétition entre les régions.

A. Univ. Prof. Dr. Ernst Steinicke, Institut für Geographie, Universität Innsbruck, Innrain 52, A-6020 Innsbruck.

e-mail:Ernst.Steinicke@uibk.ac.at

Manuskripteingang/receivend/manuscrit entré le 27.7.2001

Annahme zum Druck/accepted for publication/accepté pour l'impression: 14.12.2001 